\title{
Rituximab treatment in adults with refractory minimal change disease or focal segmental glomerulosclerosis
}

\author{
Hong Ren ${ }^{1}$, Li Lin ${ }^{1}$, Pingyan Shen ${ }^{1}, X_{i a o}$ Li $^{1}$, Jingyuan Xie ${ }^{1}, X$ iaoxia Pan ${ }^{1}$, Wen \\ Zhang ${ }^{1}$ and Nan Chen ${ }^{1}$ \\ ${ }^{1}$ Department of Nephrology, Institute of Nephrology, Ruijin Hospital, School of Medicine, Shanghai Jiaotong University, \\ Shanghai, China \\ Correspondence to: Nan Chen, email: cnri100@126.com
}

Keywords: rituximab; steroid-dependent; refractory FSGS; refractory MCD; clinical study, Immunology and Microbiology Section; Immune response; Immunity

Received: September 02, 2016 Accepted: September 05, $2017 \quad$ Published: October 15, 2017

Copyright: Ren et al. This is an open-access article distributed under the terms of the Creative Commons Attribution License 3.0 (CC BY 3.0), which permits unrestricted use, distribution, and reproduction in any medium, provided the original author and source are credited.

\section{ABSTRACT}

Rituximab (RTX) may benefit patients with glomerular disease who suffer from focal segmental glomerular sclerosis (FSGS) or minimal change disease (MCD). Here, we have described our experience treating 6 FSGS and 9 MCD patients with steroiddependent/refractory nephrotic syndrome (NS) with RTX. Patients received RTX ( $375 \mathrm{mg} / \mathrm{m2}$ ) intravenously on days 1, 8, 23, and 29. During a median follow-up of 8 months (range, 3-36 months) after RTX administration, all patients achieved complete or partial remission. Relapses decreased by approximately $\mathbf{3 0}$-fold compared with the year preceding RTX treatment, and an $89.27 \%$ reduction in proteinuria was observed. Furthermore, RTX treatment could decrease medical costs by $76.52 \%$ compared with the costs associated with the long-term use (for 12-13 months) of steroids and immunosuppressive drugs.

In conclusion, RTX treatment was safe and effective for patients with refractory FSGS or MCD.

\section{INTRODUCTION}

Minimal change disease (MCD) and focal segmental glomerular sclerosis (FSGS) are common causes of nephrotic syndrome (NS)[1, 2]. MCD accounts for $46.9 \%$ of the pediatric and $25.3 \%$ of the adult cases of primary NS in China $[1,2]$, In the USA, approximately $50-60 \%$ of adults with FSGS have NS [3, 4]. In MCD, initial therapy with oral steroids leads to a response rate of approximately $75 \%$, but a high proportion of steroidresponsive patients experiences one or more relapses of NS [2]. In FSGS, up to $63 \%$ of patients treated with steroids achieve remission. Among those achieving complete or partial remission approximately 50\% experience one or more relapses [5, 6]. Comorbidities secondary to steroid treatment may result in therapeutic dilemmas. Long-term complications of steroid therapy, such as reduced bone mineral density, dyslipidemia, embolism, impaired glucose tolerance, hypertension, and an increased risk of cardiovascular events, are commonly observed [7]. The aim of minimization of the cumulative steroid dosage, however, necessitates the use of alternative immunosuppressive agents. Despite the growing number of immunosuppressive agents that have proved to be effective, some severe cases remain difficult to treat and can lead to persistent relapsing NS. Immunosuppressive drugs including tacrolimus (TAC), cyclosporine (CsA), mycophenloatemofetil (MMF), and cyclophosphamide (CTX), are commonly used to lower or withdraw steroids in these patients.

The possibility of an effective and safe approach for patients with steroid-resistant NS (SRNS) or frequently relapsing NS (FRNS) emerged in 2004, when the B-celldepleting rituximab (RTX) was reported to have induced the remission of proteinuria in a child with FRNS secondary to MCD $[8,9]$. Moreover, RTX is a valuable option in the treatment of several connective tissue diseases and glomerular diseases such as idiopathic membranous nephropathy (IMN) [10-12]. In a small number of cases, adult patients with MCD and FSGS achieved remission after RTX treatment, suggesting that B-cell immunity could play a key role in the pathophysiologies of these diseases $[13,14]$. Several mechanisms of RTX action have been suggested, including antibody-dependent/cellmediated cytotoxicity, elimination by phagocytosis or 
cytokine-mediated cytotoxicity [15]. Nevertheless, in adult patients with MCD/FSGS, RTX was well tolerated and no life-threatening complications have been reported to date $[8,13]$. However, no study has examined RTX treatment of adult Chinese patients with MCD or FSGS. Given that China is a developing country, the use of RTX to treat kidney diseases has not been incorporated into health care in China; therefore, this study investigated the therapeutic effects of RTX in patients with refractory MCD or FSGS.

\section{RESULTS}

\section{Patient characteristics}

At study entry, 15 patients who were 16-54 years of age (median, 25 years) were enrolled, with a female-tomale ratio of 0.36 . In total, 9 patients had biopsy-proven MCD, and 6 patients had FSGS. All patients were severely nephrotic, with a time of relapse of $4 \pm 1.6$, and the average disease duration was 76months(range, 12-156 months) (Table 1). All patients had failed prior immunosuppressive treatment with a combination of steroid and CTX $(n=9)$, a combination of steroid and TAC $(n=11)$, a combination of steroid and CsA $(n=9)$, a combination of steroid and MMF ( $n=4)$, a combination of steroid and azathioprine $(n=1)$, and/or multi-targeted therapy $(n=7)$ (Table 1$)$. In this study, 15 patients received RTX therapy, including 8 patients who received RTX in combination with lowdose steroids and TAC, 2 patients who received RTX in combination with steroids and CsA, 3 patients who received RTX in combination with steroids and MMF, 1 patient who received RTX in combination with steroids and azathioprine, and 1 patient who received RTX in combination with steroids.

\section{Clinical outcomes}

During the study period, 15 patients were followed for a median duration of 8 months (range, 3-36 months). The time course of urinary protein excretion, which was $1.567 \mathrm{~g} / 24 \mathrm{~h}$ (range, $0.048-5.758 \mathrm{~g} / 24 \mathrm{~h}$ ) at baseline. By the end of 3 months, CR was achieved in 13 patients, and PR was achieved in 2 patients. The mean decrease in proteinuria from baseline to 3 months was $0.223 \mathrm{~g} / 24 \mathrm{~h}$, a reduction of $85.76 \%$. The decrease in proteinuria was gradual and stable. The mean drop in proteinuria from baseline to 6 months was $0.158 \mathrm{~g} / 24 \mathrm{~h}$, a reduction of $89.91 \%$. Among the 5 patients who completed 12 months of follow-up, 2 patients relapsed; these relapses occurred at 8 and 10 months of follow-up, with 24-hour proteinuria levels of $7.09 \mathrm{~g} / 24 \mathrm{~h}$ and $14.369 \mathrm{~g} / 24 \mathrm{~h}$, respectively. Two patients achieved CR after 2 intravenous treatments with RTX $\left(375 \mathrm{mg} / \mathrm{m}^{2}\right)$, and their mean decrease in proteinuria from baseline to 12 months was $0.186 \mathrm{~g} / 24 \mathrm{~h}$, a reduction of $89.27 \%$. Serum albumin was significantly increased among patients who achieved complete or partial remission. The remission rate of these patients at followup was $75-100 \%$ (Table 2). Based on a comparison of the year before RTX treatment and the first year of followup, during follow-up, total relapses decreased from 4 to 0.13 , and the median number of relapses decreased to 0 . Relapses decreased by 30-fold during the year of followup compared with the year preceding RTX treatment. No serious treatment-related adverse events were observed during follow-up.

\section{Steroid dosages before and after treatment with RTX}

After RTX treatment, 5 patients stopped using steroids and immunosuppressive drugs for 6 months. Thus, 5 patients reached the main endpoint, and 10 patients reached the second endpoint. The median perpatient maintenance steroid dose decreased from 25.73 $\mathrm{mg} /$ day to $5.56 \mathrm{mg} /$ day. Furthermore, the mean estimated GFR decreased from 115 to $113 \mathrm{ml} / \mathrm{min} / 1.73 \mathrm{~m}^{2}(\mathrm{P}=0.01)$. Treatment was well tolerated.

\section{$\mathrm{CD19}^{+}$changes after RTX treatment}

An initial depletion of CD19 $9^{+}$B-cells was observed in all patients (a median of 196 cells $/ \mathrm{ml}$ at baseline versus 3 cells $/ \mathrm{ml}$ at day 28). By 6 months, most patients continued to have $\mathrm{CD} 19^{+} \mathrm{B}$-cell counts $<5$ cells $/ \mathrm{ml}$. However, no associations were observed between baseline proteinuria and changes in $\mathrm{CD}_{1} 9^{+}$B-cell depletion at 6 and 12 months. There were no significant changes in hemoglobin levels, total white blood cells, or platelet counts (Table 3). We found no correlations involving B-cell counts, their subsets, or changes in T-cell parameters.

\section{Health economy}

In this study, the average medical cost for the 15 patients was $\$ 64,224$ (range, $\$ 17,460$ - $\$ 142,857$ ) prior to RTX treatment; the cost of RTX treatment was $\$ 15,079$, which was much cheaper than the cost of the long-term use of steroids and immunosuppressive drugs, and represented a cost reduction of $76.52 \%$.

\section{DISCUSSION}

RTX has shown potential role in treatment of many autoimmune diseases, including systemic vasculitis and lupus erythematosis, as well sporadic cases of MCD and FSGS. This study represented the largest prospective analysis to date that evaluated RTX in the treatment of Chinese patients with FRNS with 8 months (range, 3-36 
Table 1: Main clinical and laboratory characteristics at baseline

\begin{tabular}{ll}
\hline Characteristic & \\
\hline Number (n) & 15 \\
Age (yr) & $25(16-54)$ \\
Female/male & 0.36 \\
Disease duration (months) & $76(12-156)$ \\
Relapse number (n) & $4 \pm 1.6$ \\
Oral steroids (n) & 13 \\
Cyclosporine (n) & 9 \\
Tacrolimus (n) & 11 \\
CTX (n) & 9 \\
MMF (n) & 4 \\
Azathioprine (n) & 1 \\
MCD (n) & 9 \\
FSGS (n) & 6 \\
Proteinuria, g/24 h & $1.567(0.048-5.758)$ \\
Serum albumin, g/l & $37(14-45)$ \\
Creatinine, $\mu \mathrm{mol} / \mathrm{l}$ & $63(42-78)$ \\
GFR(EPI, ml/min/1.73 $\left.\mathrm{m}^{2}\right)$ & $128(93-135)$ \\
\hline
\end{tabular}

Table 2: Clinical outcomes before and after RTX treatment

\begin{tabular}{lllllll}
\hline Time & Baseline & Month1 & Month3 & Month6 & Month9 & Month12 \\
CR(\%) & 46.67 & 66.67 & 93.33 & 93.33 & 77.5 & 100 \\
PR(\%) & 46.67 & 26.67 & 6.67 & 6.67 & 0 & 0 \\
NR(\%) & 6.67 & 6.67 & 0.00 & 0 & 12.5 & 0 \\
Adverse event(n) & 0 & 0 & 0 & 0 & 0 & 0 \\
\hline
\end{tabular}

months) of follow-up. In this investigation, we found that RTX significantly reduced the frequency of relapses in 15 patients with frequently relapsing or steroiddependent NS. Relapses decreased by approximately 30 fold during follow-up compared with the year preceding RTX treatment, and an $89.27 \%$ reduction in proteinuria was observed. The therapeutic effects of RTX in FSGS and MCD were similar. A number of reports have reported using RTX in children with NS [16-18]. RTX therapy could significantly achieve a higher rate of complete remission and reduce the occurrence of proteinuria [19]. A recent study showed that $54.5 \%(12 / 22)$ patients aged from 6.2-25 years responded to rituximab [20]. In adult FSGS and MCD, RTX reduced the number of relapses per year from $1.3(0-9)$ relapses prior to treatment compared to $0(0-2)$ after therapy [8]. The result of our study about the remission rate in frequent relapse FSGS and MCD was similar to other studies.

In general, the mode of RTX administration was not uniform. Treatment of RTX was quite variable in different studies: a single course of rituximab consisted of 1 to 4 consecutive pulses of $375 \mathrm{mg} / \mathrm{m}^{2}$ at weekly or $1000 \mathrm{mg}$ dose dose on days 1 and 15 [8, 15, 19]. After therapy, the number of relapses reduced from $1.3(0-9)$ /year to $0(0-2)$ year $(\mathrm{p}<0.001)$ and proteinuria decreased from $2.43(0$ $15) \mathrm{g} /$ day to $0(0-4.89) \mathrm{g} /$ day $(\mathrm{p}<0.001)$ [8]. Ruggenenti et al argue for administering $375 \mathrm{mg} / \mathrm{m}^{2}$, as a single dose with circulating B cells are measured 24 hours and 1 week after Rituximab administration in order to drive RTX administration [14]. Kamel El-Reshaid et al reported that treatment with 4-weekly infusions of $500 \mathrm{mg}$ resulted in amelioration of NS in 17 of the 18 patients with FSGS, but proteinuria reappeared by 8 to 12 months $[18,21]$. The protocol of RTX was that patients received RTX (375 mg/ $\mathrm{m}^{2}$ ) intravenously on days $1,8,23$ and 29 . The reason as follows: 1) We determined that the terminal half-lives of RTX were 11.5 days in patients with $\mathrm{MN}$ and 18.0 days in patients with RA [10]. Given that almost all of these patients exhibited steroid-dependent FSGS/ MCD with moderate to nephrotic-range proteinuria, we believed the pharmacokinetic effects of RTX in these patients would persist for 11.5-18 days. 2) According to a previous report, 
Table 3: Main hematology parameters in 6 patients from rituximab administration (baseline) to the end of the study

\begin{tabular}{llll}
\hline Parameter & Baseline & 3 months & 6 months \\
\hline Hemoglobin(g/l) & $145(80-170)$ & $141(80-157)$ & $148(84-170)$ \\
White blood cell count & $11.4(7.4-18.9)$ & $7.57(4.5-20)$ & $6.9(4.3-13.7)$ \\
Lymphocytes & $3.2(1.5-13.5)$ & $2.19(1.06-4.1)$ & $2.03(1.1-28.6)$ \\
$\mathrm{IgA}(\mathrm{mg} / \mathrm{dl})$ & $199(90-340)$ & $166(149-183)$ & $207(81-291)$ \\
$\mathrm{IgG}(\mathrm{mg} / \mathrm{dl})$ & $844(218-2320)$ & $790(730-851)$ & $1185(470-1650)$ \\
$\mathrm{IgM}(\mathrm{mg} / \mathrm{dl})$ & $129(22-254)$ & $89(21-157)$ & $112(28-246)$
\end{tabular}

B cell subset analysis in peripheral blood mononuclear cells

$\mathrm{CD} 19^{+}(\mathrm{B}$ cells $) \quad$ 196(16-595) $\quad$ 5.8(1-290)

$\mathrm{T}$ cell subset analysis in peripheral blood mononuclear cells

\begin{tabular}{llll}
$\mathrm{CD}^{+} \mathrm{T}$ cells $(\%)$ & $9.85(1.3-36.3)$ & $82.75(73.3-93.8)$ & $84.05(77.7-92.7)$ \\
$\mathrm{CD}^{+}{ }^{+} \mathrm{CD} 4^{+}$(helper) T cells(\%) & $78.90(7.1-83.8)$ & $39(18.6-42.2)$ & $46.45(43.6-48.2)$ \\
$\mathrm{CD}^{+} \mathrm{CD}^{+}$(suppressor) T & $37.65(5.9-55.2)$ & $42.25(35.7-54.6)$ & $34.65(31.1-38.2)$ \\
cells $(\%)$ & & & \\
\hline
\end{tabular}

RTX will be lost via urinary excretion [10], half-life period would shorter than 11.5 days. So we regarded 4-weekly consecutive pulses of $375 \mathrm{mg} / \mathrm{m} 2$ RTX ensured the drug concentration reached an appropriate peak. 3) The reasons why we prolonged interval to 2 weeks between the second RTX doses and third as followed: After 2 times treatment, patients' proteinuria decreased significantly. So less RTX leakage from the kidney and longer pharmacokinetic occur, and the interval after the second treatment can be extended to $11.5-18$ days.

The results of our study strongly support a role for RTX in the treatment of FSGS/MCD and offer a more selective therapeutic approach. We observed CR or PR in $75-100 \%$ of patients at 1 year. Consistent with previous reports, RTX was well tolerated, and infusion reactions were the most common side effects. Furthermore, this study determined that the cost of RTX treatment was no greater than that associated with the long-term use of traditional treatments for FSGS and MCD. In fact, RTX treatment could reduce spending for frequently relapsing patients.

The limitations of this study include the lack of a randomized design, the absence of a placebo control, and a limited sample size. Furthermore, follow-up beyond 3 years is required to assess the durability and safety of responses to RTX.

In conclusion, RTX appears to effectively decrease relapses, and RTX treatment can achieve CR or PR of proteinuria in patients with FSGS or MCD in a costeffective manner. Our results are particularly remarkable given that all patients had failed their therapies prior to treatment with RTX. We found that intravenous RTX
(375 mg/m $\mathrm{m}^{2}$ ) on days $1,8,23$, and 29 was an appropriate dosing approach for steroid-dependent/refractory FSGS and MCD. More prospective randomized controlled trials of RTX are needed to truly assess this drug's efficacy and durability compared with traditional specific immunosuppressive treatments.

\section{MATERIALS AND METHODS}

\section{Patient population}

This study was a single-center prospective study. Before treatment, we obtained informed consent from the included patients and approval from the scientific and ethics committee of our hospital.

We recruited 15 patients with FRNS who had renal biopsy-proven FSGS or MCD, exclude inheritance and secondary (Supplementary Figures 1-3, Supplementary Table 1), exhibited evidence of long-term treatment with immunosuppressive drugs (CsA, TAC, CTX, MMF or azathioprine), and had a GFR (glomerular filtration rate) higher than $60 \mathrm{ml} / \mathrm{min}$ calculated using the CKDEPI (chronic kidney disease epidemiology collaboration) equation. FRNS was defined as 2 or more relapses within 6 months of the initial response or 4 or more relapses in any 12-month period. The exclusion criteria were pregnancy, infections (including hepatitis $\mathrm{C}$, hepatitis B and HIV), diabetes mellitus, malignancy, glomerulonephritis other than FSGS or MCD, and any systemic disease. 


\section{Treatment protocol and follow-up}

FRNS patients with biopsy-proven FSGS or MCD were enrolled. They were treated with steroids, RTX and low-dose immunosuppressive agents. RTX (375 mg/ $\mathrm{m}^{2}$ ) was administered intravenously on days $1,8,23$ and 29. To minimize infusion reactions, patients were orally premedicated with acetaminophen and diphenhydramine hydrochloride. In addition, methylprednisolone $(40 \mathrm{mg}$, intravenous) was administered prior to the first RTX infusion. B-cell depletion was defined as a $\mathrm{CD} 19^{+}$count $<5$ cells $/ \mu 1$. One month after the final infusion of RTX, the doses of both steroids and immunosuppressive drugs were tapered each month until these drugs were successfully discontinued or evidence of a relapse of NS was observed.

Before treatment, a kidney biopsy was performed for all patients. Patients' baseline clinical characteristics and laboratory data were reviewed after the screening visit. The following data were collected and evaluated at study entry and at months 1, 3, 6, 9, and 12: clinical features, blood pressure, side effects, findings from routine examinations of blood and urine, liver and renal function, plasma electrolyte content, 24-hour protein excretion, serum cholesterol, serum glucose, and serum immunoglobulins (IgG, IgM, IgA, IgE). The medical costs associated with RTX and with other therapies used prior to RTX were also assessed for each patient to analyze whether RTX therapy was economically advantageous.

\section{Outcome measures}

The main outcome measures were the percentage of patients who achieved treatment withdrawal with no evidence of relapse and the percentage of patients who maintained complete or partial remission after treatment withdrawal. The secondary endpoints included monthly changes in protein and the number of patients with PR (partial remission) or CR (complete remission) at 6 and 12 months. CR was defined as proteinuria $<0.3 \mathrm{~g} / 24 \mathrm{~h}$, PR was defined as proteinuria $<3.5 \mathrm{~g} / 24 \mathrm{~h}$ and a $<50 \%$ reduction in peak proteinuria, and nonresponse was defined as a $<50 \%$ reduction in peak proteinuria. Any patient who achieved $\mathrm{CR}$ or PR was regarded as a treatment success.

\section{Statistical analyses}

Monthly changes were tested against zero using paired t-tests. Associations between urinary protein and immune response were assessed using Pearson's correlation coefficients for two-sided hypothesis tests performed with a significance threshold of 0.05 . Statistical analyses were conducted using SPSS, version 20.

\section{CONFLICTS OF INTEREST}

The authors disclose no potential conflicts of interest.

\section{FUNDING}

This work was supported by grants from Shanghai Jiaotong University School of Medicine multi-center clinical research program, grant no. DLY201510; National Basic Research Program of China973, grant no. 2012CB517600 (no. 2012CB517604); National Basic Research Program of Shanghai, grant no15ZR1426300; National Key Research and Development Program of China (2016YFC0904100); National Natural Science Foundation of China (No. 81570598).

\section{REFERENCES}

1. Pan X, Xu J, Ren H, Zhang W, Xu Y, Shen P, Li X, Wang W, Chen X, Wu P, Feng X, Hao C, Chen N. Changing spectrum of biopsy-proven primary glomerular diseases over the past 15 years: a single-center study in China. Contributions to nephrology. 2013; 181:22-30.

2. Xie J, Chen N. Primary glomerulonephritis in mainland China: an overview. Contributions to nephrology. 2013; 181:1-11.

3. Chun MJ, Korbet SM, Schwartz MM, Lewis EJ. Focal segmental glomerulosclerosis in nephrotic adults: presentation, prognosis, and response to therapy of the histologic variants. Journal of the American Society of Nephrology : JASN. 2004; 15:2169-2177.

4. Troyanov S, Wall CA, Miller JA, Scholey JW, Cattran DC, Toronto Glomerulonephritis Registry G. Focal and segmental glomerulosclerosis: definition and relevance of a partial remission. Journal of the American Society of Nephrology : JASN. 2005; 16:1061-1068.

5. Ren H, Shen P, Li X, Pan X, Zhang Q, Feng X, Zhang W, Chen N. Treatment and prognosis of primary focal segmental glomerulosclerosis. Contributions to nephrology. 2013; 181:109-118.

6. Ren H, Shen P, Li X, Pan X, Zhang W, Chen N. Tacrolimus versus cyclophosphamide in steroid-dependent or steroidresistant focal segmental glomerulosclerosis: a randomized controlled trial. Am J Nephrol. 2013; 37:84-90.

7. Gbadegesin R, Lavin P, Foreman J, Winn M. Pathogenesis and therapy of focal segmental glomerulosclerosis: an update. Pediatr Nephrol. 2011; 26:1001-1015.

8. Kronbichler A, Kerschbaum J, Fernandez-Fresnedo G, Hoxha E, Kurschat CE, Busch M, Bruchfeld A, Mayer G, Rudnicki M. Rituximab treatment for relapsing minimal change disease and focal segmental glomerulosclerosis: a systematic review. Am J Nephrol. 2014; 39:322-330.

9. Bagga A, Sinha A, Moudgil A. Rituximab in patients with 
the steroid-resistant nephrotic syndrome. The New England journal of medicine. 2007; 356:2751-2752.

10. Fervenza FC, Cosio FG, Erickson SB, Specks U, Herzenberg AM, Dillon JJ, Leung N, Cohen IM, Wochos DN, Bergstralh E, Hladunewich M, Cattran DC. Rituximab treatment of idiopathic membranous nephropathy. Kidney international. 2008; 73:117-125.

11. Fervenza FC, Abraham RS, Erickson SB, Irazabal MV, Eirin A, Specks U, Nachman PH, Bergstralh EJ, Leung N, Cosio FG, Hogan MC, Dillon JJ, Hickson LJ, et al. Rituximab therapy in idiopathic membranous nephropathy: a 2-year study. Clinical journal of the American Society of Nephrology : CJASN. 2010; 5:2188-2198.

12. Ruggenenti P, Cravedi P, Chianca A, Perna A, Ruggiero B, Gaspari F, Rambaldi A, Marasa M, Remuzzi G. Rituximab in idiopathic membranous nephropathy. Journal of the American Society of Nephrology : JASN. 2012; 23:14161425.

13. Iwabuchi $Y$, Takei $T$, Moriyama $T$, Itabashi M, Nitta $\mathrm{K}$. Long-term prognosis of adult patients with steroiddependent minimal change nephrotic syndrome following rituximab treatment. Medicine (Baltimore). 2014; 93:e300.

14. Ruggenenti P, Ruggiero B, Cravedi P, Vivarelli M, Massella L, Marasa M, Chianca A, Rubis N, Ene-Iordache B, Rudnicki M, Pollastro RM, Capasso G, Pisani A, et al. Rituximab in steroid-dependent or frequently relapsing idiopathic nephrotic syndrome. Journal of the American Society of Nephrology : JASN. 2014; 25:850-863.

15. Kattah AG, Fervenza FC, Roccatello D. Rituximab-based novel strategies for the treatment of immune-mediated glomerular diseases. Autoimmunity reviews. 2013; 12:854859.
16. Ravani P, Magnasco A, Edefonti A, Murer L, Rossi R, Ghio L, Benetti E, Scozzola F, Pasini A, Dallera N, Sica F, Belingheri M, Scolari F, Ghiggeri GM. Short-Term Effects of Rituximab in Children with Steroid- and CalcineurinDependent Nephrotic Syndrome: A Randomized Controlled Trial. Clinical journal of the American Society of Nephrology : CJASN. 2011; 6: 1308-1315.

17. Magnasco A, Ravani P, Edefonti A, Murer L, Ghio L, Belingheri M, Benetti E, Murtas C, Messina G, Massella L, Porcellini MG, Montagna M, Regazzi M, et al. Rituximab in Children with Resistant Idiopathic Nephrotic Syndrome. Journal of the American Society of Nephrology : JASN. 2012; 23: 1117-1124.

18. El-Reshaid K, Sallam HT, Hakim AA, Al-Attiyah R. Rituximab as a Rescue Therapy in Patients with Glomerulonephritis. Saudi J Kidney Dis Transpl. 2012; 23:973-978.

19. Zhao Z, Liao G, Li Y, Zhou S, Zou H. The efficacy and safety of rituximab in treating childhood refractory nephrotic syndrome: A meta-analysis. Sci Rep. 2014; 5:8219.

20. Chan CY, Liu ID, Resontoc LP, Ng KH, Chan YH, Lau PY, Than M, Jordan SC, Lam KP, Yeo WS, Yap HK. $\mathrm{T}$ Lymphocyte Activation Markers as Predictors of Responsiveness to Rituximab among Patients with FSGS. Clin J Am Soc Nephrol. 2016; 11:1360-1368.

21. El-Reshaid K, Sallam HT, Hakim AA, Al-Attiyah R. Rituximab in Treatment of Idiopathic Glomerulopathy. Saudi J Kidney Dis Transpl. 2012; 23:973-978. 